\title{
Erratum: Dimension-six matrix elements for meson mixing and lifetimes from sum rules
}

\section{Kirk, A. Lenz and T. Rauh}

IPPP, Department of Physics, University of Durham, DH1 3LE, United Kingdom

E-mail: m.j.kirk@durham.ac.uk, alexander.lenz@durham.ac.uk, thomas.j.rauh@durham.ac.uk

ERRATUM TO: JHEP12(2017)068

ARXIV EPRINT: 1711.02100

Eq. (5.15) in the original paper used a wrong numerical expression for the lifetime ratio $\tau\left(B_{s}^{0}\right) / \tau\left(B_{d}^{0}\right)$ from [16] and should read

$$
\begin{aligned}
\left.\frac{\tau\left(B_{s}^{0}\right)}{\tau\left(B^{0}\right)}\right|_{\exp } & =0.994 \pm 0.004 \\
\left.\frac{\tau\left(B_{s}^{0}\right)}{\tau\left(B^{0}\right)}\right|_{\overline{\mathrm{MS}}} & =1.0007 \pm 0.0025 \\
& =1.0007 \pm 0.0014 \text { (had.) } \pm 0.0006 \text { (scale) } \pm 0.0020\left(1 / m_{b}^{4}\right),
\end{aligned}
$$

implying an agreement with experiment at the level of 1.4 instead of $1.1 \sigma$ as stated originally. Figure 9 is also affected and is updated below.

In the original paper we have set color factors in some places to their QCD values with $N_{c}=3$ while keeping them general in others. ${ }^{1}$ To restore generality one has to change the following eqs. to

$$
\begin{aligned}
\Delta \rho_{\tilde{Q}_{i}} & \equiv \frac{N_{c} C_{F}}{4} \frac{\omega_{1}^{2} \omega_{2}^{2}}{\pi^{4}} \frac{\alpha_{s}}{4 \pi} r_{\tilde{Q}_{i}}\left(x, L_{\omega}\right), \\
w_{\tilde{Q}_{i}}\left(\omega_{1}, \omega_{2}\right) & =\frac{\Delta \rho_{\tilde{Q}_{i}}^{\text {pert }}\left(\omega_{1}, \omega_{2}\right)}{\rho_{\Pi}^{\text {pert }}\left(\omega_{1}\right) \rho_{\Pi}^{\text {pert }}\left(\omega_{2}\right)}=\frac{C_{F}}{N_{c}} \frac{\alpha_{s}}{4 \pi} r_{\tilde{Q}_{i}}\left(x, L_{\omega}\right), \\
\Delta B_{\tilde{Q}_{i}}^{\text {pert }}\left(\mu_{\rho}\right) & =\frac{C_{F}}{N_{c} A_{\tilde{Q}_{i}}} \frac{\alpha_{s}\left(\mu_{\rho}\right)}{4 \pi} r_{\tilde{Q}_{i}}\left(1, \log \frac{\mu_{\rho}^{2}}{4 \bar{\Lambda}^{2}}\right),
\end{aligned}
$$

which coincide with their counterparts in the original paper in QCD with three colors.

\footnotetext{
${ }^{1}$ We are grateful to A. Grozin and A. Pivovarov for pointing this out to us.
} 
We noticed that the labels "This work $\left(f_{D}^{\text {lattice }}\right)$ " and "This work $\left(f_{D}^{\exp }\right)$ " in figure 7 were interchanged. The figure with the corrected labels in shown below.

Our numerical code for $\Delta M_{q}$ and $a_{\mathrm{sl}}^{q}$ used a wrong input value for the top-quark mass. ${ }^{2}$ The corresponding SM predictions in eqs. (4.4), (4.5), (4.7) and (4.8) change by a small amount to

$$
\begin{aligned}
\Delta M_{s}^{\mathrm{SM}} & \left.=(16.6 \pm 1.7) \mathrm{ps}^{-1}=\left(16.6_{-1.1}^{+1.2} \text { (had. }\right) \pm 0.1(\text { scale })_{-1.2}^{+1.3}(\text { param. })\right) \mathrm{ps}^{-1} \\
\Delta M_{d}^{\mathrm{SM}} & =(0.56 \pm 0.08) \mathrm{ps}^{-1}=(0.56 \pm 0.04(\text { had. }) \pm 0.00(\text { scale }) \pm 0.07(\text { param. })) \mathrm{ps}^{-1} \\
a_{\mathrm{sl}}^{s, \overline{\mathrm{MS}}} & \left.=(2.3 \pm 0.3) \cdot 10^{-5}=(2.3 \pm 0.1 \text { (had. })_{-0.1}^{+0.0}(\text { scale }) \pm 0.3(\text { param. })\right) \cdot 10^{-5} \\
a_{\mathrm{sl}}^{s, \mathrm{PS}} & \left.=(2.2 \pm 0.3) \cdot 10^{-5}=(2.2 \pm 0.1 \text { (had. })_{-0.1}^{+0.0}(\text { scale })_{-0.3}^{+0.2}(\text { param. })\right) \cdot 10^{-5} \\
a_{\mathrm{sl}}^{s, 1 \mathrm{~S}} & \left.=\left(2.1_{-0.3}^{+0.2}\right) \cdot 10^{-5}=(2.1 \pm 0.1 \text { (had. })_{-0.1}^{+0.0}(\text { scale })_{-0.3}^{+0.2}(\text { param. })\right) \cdot 10^{-5} \\
a_{\mathrm{sl}}^{s, \mathrm{kin}} & \left.=(2.2 \pm 0.3) \cdot 10^{-5}=(2.2 \pm 0.1 \text { (had. })_{-0.1}^{+0.0}(\text { scale })_{-0.3}^{+0.2}(\text { param. })\right) \cdot 10^{-5} \\
a_{\mathrm{sl}}^{d, \mathrm{PS}} & =\left(-4.4_{-0.5}^{+0.6}\right) \cdot 10^{-4}=\left(-4.4 \pm 0.1(\text { had. })_{-0.1}^{+0.2}(\text { scale }) \pm 0.5(\text { param. })\right) \cdot 10^{-4}
\end{aligned}
$$

which improves the agreement of $\Delta M_{d}^{\mathrm{SM}}$ with the experimental value to better than one standard deviation. This also affects figure 8 as well as tables 6 and 7 which are corrected below. All our conclusions remain unchanged.

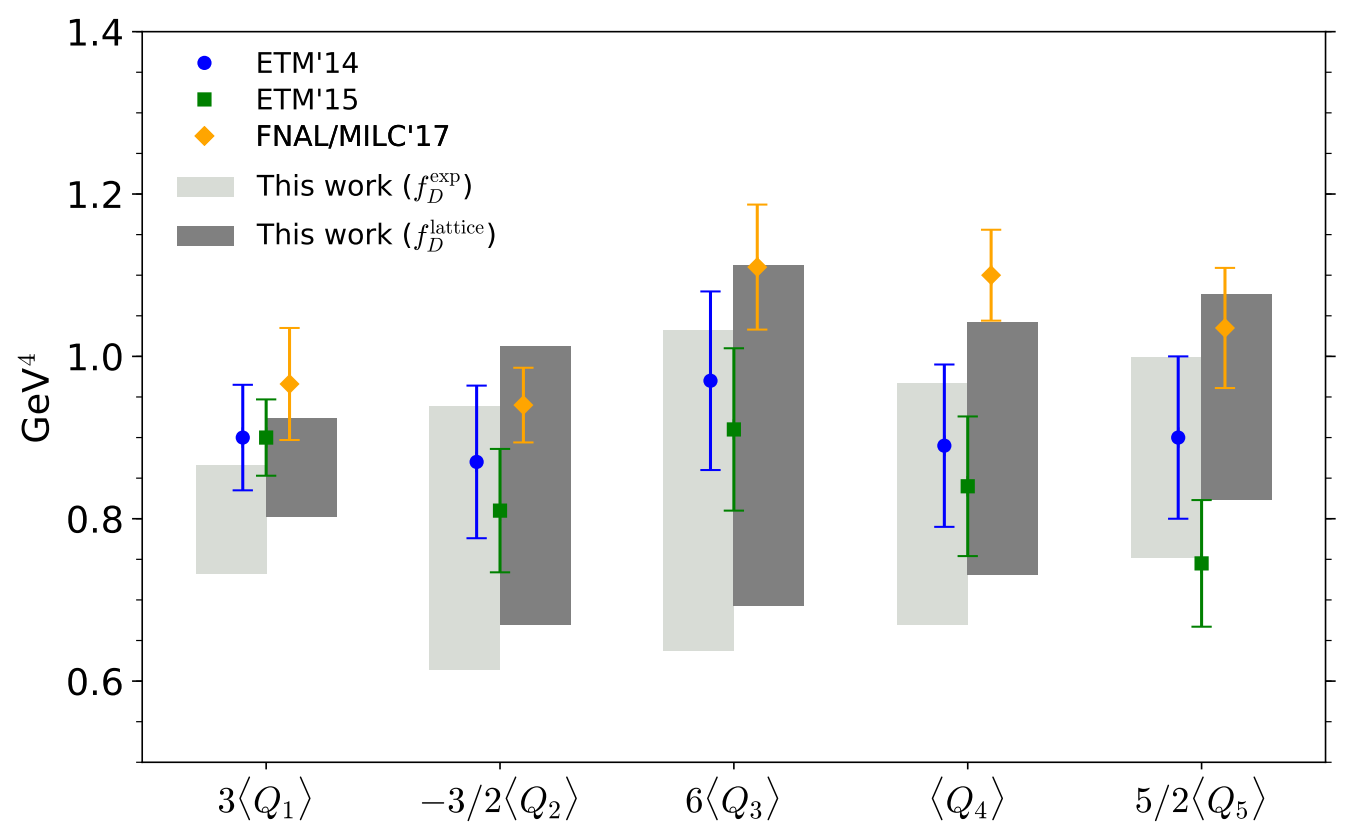

Figure 7. Comparison of our results for the $\Delta C=2$ matrix elements at the scale $3 \mathrm{GeV}$ to the lattice values of ETM'14 [30], ETM'15 [31] and FNAL/MILC'17 [32] The values for the matrix elements of the ETM collaboration are extracted from figure 16 of [32].

\footnotetext{
${ }^{2}$ We wish to thank G. Tetlalmatzi-Xolocotzi for pointing this out to us.
} 

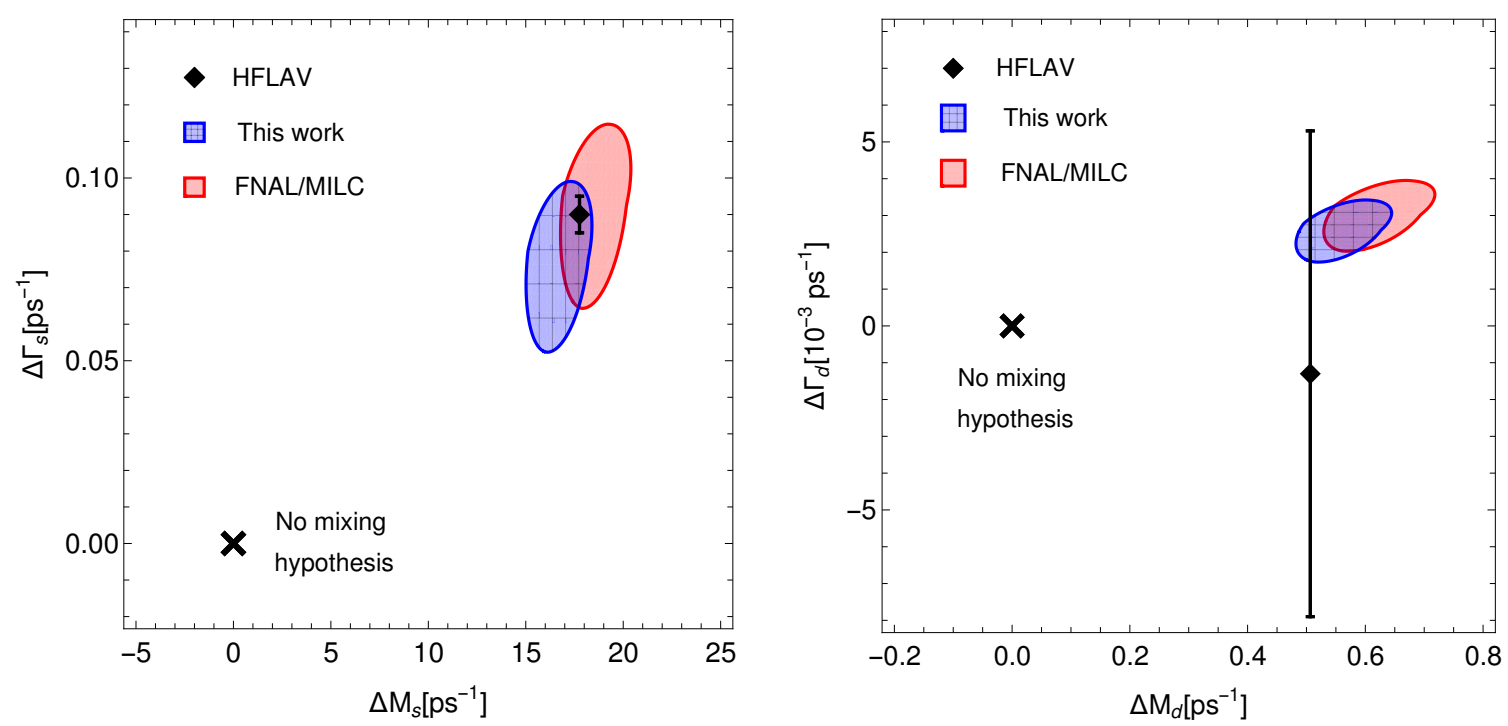

Figure 8. Comparison of our predictions for the mass and decay rate difference in the $B_{s}$ (left) and $B_{d}$ (right) system with the present experimental averages (error bars). We also show the results obtained with the lattice results of [5] for $f_{B_{q}}^{2} \bar{B}_{Q_{i}}$ and the matrix element $\left\langle R_{0}\right\rangle$ and the values given in appendix B for the other input parameters. The PS mass scheme for the bottom quarks has been used in both cases.

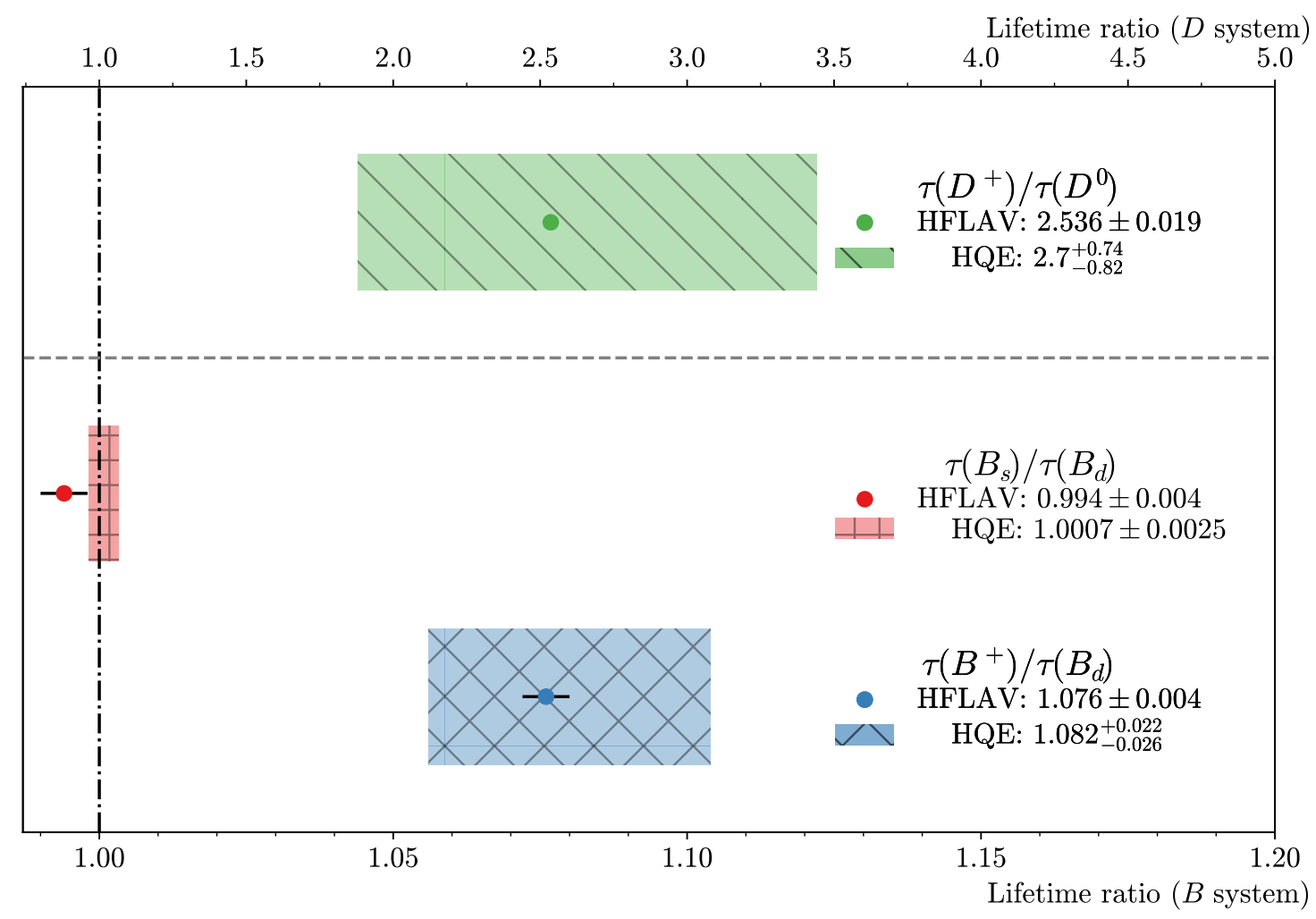

Figure 9. Comparison of our predictions for the lifetime ratios of heavy mesons with the present experimental averages. 


\begin{tabular}{|l|ccc|}
\hline & $\Delta M_{s}^{\mathrm{SM}}\left[\mathrm{ps}^{-1}\right]$ & $\Delta \Gamma_{s}^{\mathrm{PS}}\left[\mathrm{ps}^{-1}\right]$ & $a_{\mathrm{sl}}^{s, \mathrm{PS}}\left[10^{-5}\right]$ \\
\hline $\bar{B}_{Q_{1}}$ & \pm 1.0 & \pm 0.005 & \pm 0.01 \\
$\bar{B}_{Q_{3}}$ & \pm 0.0 & \pm 0.005 & \pm 0.02 \\
$\bar{B}_{R_{0}}$ & \pm 0.0 & \pm 0.003 & \pm 0.00 \\
$\bar{B}_{R_{1}}$ & \pm 0.0 & \pm 0.000 & \pm 0.00 \\
$\bar{B}_{R_{1}^{\prime}}$ & \pm 0.0 & \pm 0.000 & \pm 0.00 \\
$\bar{B}_{R_{2}}$ & \pm 0.0 & \pm 0.016 & \pm 0.00 \\
$\bar{B}_{R_{3}}$ & \pm 0.0 & \pm 0.000 & \pm 0.02 \\
$\bar{B}_{R_{3}^{\prime}}$ & \pm 0.0 & \pm 0.000 & \pm 0.05 \\
$f_{B_{s}}$ & \pm 0.5 & \pm 0.002 & \pm 0.00 \\
$\mu_{1}$ & \pm 0.0 & ${ }_{-0.018}^{+0.007}$ & ${ }_{-0.09}^{+0.04}$ \\
$\mu_{2}$ & \pm 0.1 & ${ }_{-0.002}^{+0.000}$ & ${ }_{-0.01}^{+0.02}$ \\
$m_{b}$ & \pm 0.0 & ${ }_{-0.001}^{+0.000}$ & ${ }_{-0.01}^{+0.02}$ \\
$m_{c}$ & \pm 0.0 & ${ }_{-0.000}^{+0.000}$ & \pm 0.07 \\
$\alpha_{s}$ & \pm 0.0 & \pm 0.000 & \pm 0.04 \\
$\mathrm{CKM}$ & ${ }_{-1.2}^{+1.3}$ & \pm 0.006 & ${ }_{-0.24}^{+0.23}$ \\
\hline
\end{tabular}

Table 6. Individual errors for the $B_{s}$ mixing observables.

\begin{tabular}{|l|ccc|}
\hline & $\Delta M_{d}^{\mathrm{SM}}\left[\mathrm{ps}^{-1}\right]$ & $\Delta \Gamma_{d}^{\mathrm{PS}}\left[10^{-3} \mathrm{ps}^{-1}\right]$ & $a_{\mathrm{sl}}^{d, \mathrm{PS}}\left[10^{-4}\right]$ \\
\hline $\bar{B}_{Q_{1}}$ & \pm 0.03 & \pm 0.16 & \pm 0.02 \\
$\bar{B}_{Q_{3}}$ & \pm 0.00 & +0.17 & \pm 0.03 \\
$\bar{B}_{R_{0}}$ & \pm 0.00 & \pm 0.11 & \pm 0.01 \\
$\bar{B}_{R_{1}}$ & \pm 0.00 & \pm 0.01 & \pm 0.00 \\
$\bar{B}_{R_{1}^{\prime}}$ & \pm 0.00 & \pm 0.01 & \pm 0.00 \\
$\bar{B}_{R_{2}}$ & \pm 0.00 & \pm 0.54 & \pm 0.00 \\
$\bar{B}_{R_{3}}$ & \pm 0.00 & \pm 0.00 & \pm 0.04 \\
$\bar{B}_{R_{3}^{\prime}}$ & \pm 0.00 & \pm 0.01 & \pm 0.10 \\
$f_{B}$ & \pm 0.02 & \pm 0.11 & \pm 0.00 \\
$\mu_{1}$ & \pm 0.00 & ${ }_{-0.62}^{+0.24}$ & ${ }_{-0.08}^{+0.19}$ \\
$\mu_{2}$ & \pm 0.00 & ${ }_{-0.08}^{+0.00}$ & ${ }_{-0.03}^{+0.01}$ \\
$m_{b}$ & \pm 0.00 & ${ }_{-0.03}^{+0.01}$ & ${ }_{-0.03}^{+0.01}$ \\
$m_{c}$ & \pm 0.00 & \pm 0.02 & \pm 0.14 \\
$\alpha_{s}$ & \pm 0.00 & \pm 0.01 & ${ }_{-0.08}^{+0.09}$ \\
$\mathrm{CKM}$ & \pm 0.07 & $+{ }_{-0.37}^{+0.36}$ & \pm 0.49 \\
\hline
\end{tabular}

Table 7. Individual errors for the $B_{d}$ mixing observables. 
Open Access. This article is distributed under the terms of the Creative Commons Attribution License (CC-BY 4.0), which permits any use, distribution and reproduction in any medium, provided the original author(s) and source are credited. 